\title{
The Construction of Polygenic Risk Scores for Breast Cancer Based on LightGBM and Multiple Omics Data
}

\section{Baoshan Ma ( $\nabla$ mabaoshan@dlmu.edu.cn )}

Dalian Maritime University https://orcid.org/0000-0003-1629-1968

Jianqiao Pan

Dalian Maritime University

\section{Xiaoyu Hou}

Dalian Maritime University

Chongyang Li

Dalian Maritime University

\section{Tong Xiong}

Dalian Maritime University

\section{Yi Gong}

Dalian Maritime University

\section{Fengju Song}

Tianjin Medical University Cancer Institute and Hospital: Tianjin Tumor Hospital

\section{Research Article}

Keywords: Breast cancer, Polygenic risk scores, Multiple omics data, LightGBM, Diagnosis, Prognosis

Posted Date: April 30th, 2021

DOl: https://doi.org/10.21203/rs.3.rs-438740/v1

License: (a) This work is licensed under a Creative Commons Attribution 4.0 International License. Read Full License 


\section{The Construction of Polygenic Risk Scores for Breast Cancer Based on LightGBM and Multiple Omics Data}

1 Baoshan Ma ${ }^{1, *}$, Jianqiao Pan ${ }^{1}$, Xiaoyu Hou ${ }^{1}$, Chongyang Li $^{1}$, Tong Xiong ${ }^{1}$, Yi Gong ${ }^{1}$,

2 Fengju Song, ${ }^{2, *}$

$3{ }^{1}$ School of Information Science and Technology, Dalian Maritime University, Dalian,

4 116026, China

$5 \quad{ }^{2}$ Department of Epidemiology and Biostatistics, Key Laboratory of Molecular Cancer

6 Epidemiology, Tianjin, National Clinical Research Center of Cancer, Tianjin Medical

7 University Cancer Institute and Hospital, Tianjin, 300060, China

8 *Corresponding author:

9 E-mail addresses: mabaoshan@dlmu.edu.cn (Baoshan Ma), songfengju@163.com (Fengju

10 Song)

11 Tel: +86 18624392829 (Baoshan Ma), +86 13164066716 (Fengju Song)

12 


\section{Abstract}

20 Background: Breast cancer accounts for a large proportion of cancer-related deaths in

21 women. Polygenic risk score (PRS) derived from single nucleotide polymorphisms (SNP)

22 data can evaluate the individual-level genetic risk of breast cancer and has been widely

23 applied for risk stratification. However, standalone SNP data used for PRS may not provide

24 satisfactory prediction accuracy. Additionally, current PRS models based on linear regression

25 have insufficient power to leverage non-linear effects from thousands of associated SNPs.

26 Methods: In this study, the multiple omics data (DNA methylation data, miRNA data, mRNA data and lncRNA data) and clinical data of breast invasive carcinoma (BRCA) were collected from The Cancer Genome Atlas (TCGA). First, we developed a novel PRS model utilizing single omic data and a machine learning algorithm (LightGBM). Subsequently, we built a combination model of PRS derived from each omic data to explore whether multiple omics

31 data can further improve the prediction accuracy of PRS. Finally, we performed association analysis and prognosis prediction of breast cancer to evaluate the utility of the PRS generated by our method.

Results: Our PRS model based on single omic data and LightGBM algorithm achieved better predictive performance than the linear models and other machine learning models. Moreover, the combination of the PRS derived from each omic data can efficiently strengthen prediction accuracy. The analysis of prevalence and the associations of the PRS with phenotypes including case-control and cancer stage status indicated that the risk of breast cancer increases 
39 with the increases of PRS. The survival analysis also suggested that PRS for the cancer stage

40 is an effective prognostic metric of breast cancer patients.

41 Conclusion: Our proposed model expanded the current definition of PRS from standalone

42 SNP data to multiple omics data and outperformed the state-of-the-art PRS models, which

43 may provide a powerful tool for diagnostic and prognostic prediction of breast cancer.

44 Keywords: Breast cancer, Polygenic risk scores, Multiple omics data, LightGBM, Diagnosis,

45 Prognosis

46

47

48

49

50

51

52

53

54

55

56

57

58

59

60 


\section{Introduction}

Breast cancer is the most frequently diagnosed cancer in women worldwide[1]. In 2020, there were over 2 million new cases reported[2]. The establishment of effective prevention and treatment measures is essential to prevent breast cancer occurrence and reduce breast cancer mortality. Although carriers of BRCA1 and BRCA2 gene mutations confer a high risk of breast cancer, these gene mutations can be found in only a small part of breast cancer patients[3]. In recent years, genome-wide association study (GWAS) identified multiple high frequency and low penetrance susceptibility variants of breast cancer[4]. The accumulation effects of these susceptibility variants can be summarized as a polygenic risk score (PRS). In recent years, researchers have developed several PRS models for breast cancer by using a large amount of single nucleotide polymorphisms (SNPs) data. These studies maintained the PRS to be an effective and reliable predictor of breast cancer risk that may provide screening and prevention strategies[5-9].

However, the PRS calculated using SNPs data can only assess the genetic risk of an individual, while ignoring the influence of the external environmental exposure on gene expression. With the development of high-throughput omics technology, a large number of related studies based on genomics and transcriptomics emerged[10, 11]. These high-throughput molecular markers can dynamically reflect the comprehensive effects of genetic background, environmental exposure and lifestyle habits individually[12-14]. The analyses of multiple omics data may lead to new insights into diagnosis and prognosis of 
81 breast cancer[15]. In addition, in the standard approach of PRS, the effect sizes of the genetic

82 variants are usually estimated in linear statistical models[16-19]. However, linear statistical

83 model has some limitations and only be applied when specific requirements are satisfied[20].

84 Advanced machine learning (ML) models[21, 22] such as LightGBM can account for

85 non-linear relationships among large-scale variables and have an increasing trend on the

86 applications for breast cancer research. Using these ML models may further improve the

87 prediction accuracy (PA) of PRS.

88 Here, we used multiple omics data and LightGBM model to construct a novel PRS for breast

89 cancer. The results illustrated that our proposed method outperforms traditional linear models

90 and other state-of-the-art ML models and can effectively predict individual risk of breast

91 cancer.

92 2. Methods

\subsection{Data collection}

94 The datasets in this study were downloaded from The Cancer Genome Atlas (TCGA) project.

95 Now, all TCGA data are accessible without limitations in publications or presentations

96 according to the posted announcement from the TCGA website[23]. We collected four kinds of

97 omics datasets on breast invasive carcinoma (BRCA), including DNA methylation data

98 (Illumina Infinium Human DNA Methylation 450K; Level 3) measured from 782 tumor tissues

99 and 96 normal tissues (Paracancerous tissue), miRNA-seq data (IlluminaHiSeq miRNASeq;

100 Level 3) measured from 1078 tumor tissues and 104 normal tissues, mRNA expression 
101 (Illumina mRNA-seq; Level 3) measured from 1102 tumor tissues and 113 normal tissues,

102 IncRNA expression (Illumina lncRNA-seq; Level 3) measured from 1102 tumor tissues and

103113 normal tissues. We also collected the stage of tumor for the BRCA patients, including

104 stage I, stage II, stage III, and stage IV. According to the literature[24], the annotation of stage I

105 and II were labelled as early-stage, stage III and IV as late-stage. For BRCA patients, most of

106 the individuals are white, and a small number of individuals are black or African American

107 and Asian. Although the female patients of breast cancer account for the majority, there are

108 also 5 male patients in the BRCA datasets. The ages of volunteers used in our study range

109 from 26 to 90 . Table 1 and Supplementary Figure 1 shows the number of sampels in BRCA

110 datasets and the detail of basic demographic characteristics, respectively.

Table 1. The description of datasets used in this study.

\begin{tabular}{|c|c|c|c|c|c|}
\hline Omic type & $\begin{array}{r}\text { Total of early-s } \\
\text { late-stage } t \\
\text { sample }\end{array}$ & $\begin{array}{l}\text { or and } \\
\text { or }\end{array}$ & $\begin{array}{l}\text { Total of tumor } \\
\text { samples }\end{array}$ & $\begin{array}{l}\text { Total of } \\
\text { normal } \\
\text { samples }\end{array}$ & $\begin{array}{c}\text { Total of } \\
\text { biological } \\
\text { variables }\end{array}$ \\
\hline $\begin{array}{c}\text { DNA } \\
\text { methylation }\end{array}$ & $\begin{array}{l}\text { Early-stage } \\
\text { Late-stage }\end{array}$ & $\begin{array}{l}562 \\
209\end{array}$ & 782 & 96 & 14797 \\
\hline miRNA & $\begin{array}{l}\text { Early-stage } \\
\text { Late-stage }\end{array}$ & $\begin{array}{l}790 \\
264\end{array}$ & 1078 & 104 & 360 \\
\hline mRNA & $\begin{array}{l}\text { Early-stage } \\
\text { Late-stage }\end{array}$ & $\begin{array}{l}800 \\
267\end{array}$ & 1102 & 113 & 16499 \\
\hline $\operatorname{lncRNA}$ & $\begin{array}{l}\text { Early-stage } \\
\text { Late-stage }\end{array}$ & $\begin{array}{l}800 \\
267\end{array}$ & 1102 & 113 & 5382 \\
\hline
\end{tabular}

The total of tumor samples is not equal to the sum of the early-stage and the late-stage samples,

111 because some tumor samples have unknown breast cancer stage.

\section{$112 \quad 2.2$ Data pre-processing}


113 For DNA methylation, we retained the CpG sites that most negatively correlated with gene

114 expression according to Firehose[25] and removed $\mathrm{CpG}$ sites with missing value to ensure the

115 quality of the datasets[26]. For miRNA, mRNA, and lncRNA, two steps were performed to

116 deal with the missing values in the datasets[27]. First, the probes were excluded if there is

117 missing value in more than $20 \%$ of samples. Second, all data were normalized by Min-Max

118 scaling to map the range from 0 to 1 . For convenience, $\mathrm{CpG}$ sites of DNA methylation and

119 probes of miRNA, mRNA and lncRNA are collectively referred to as biological variables.

120 Table 1 also shows the the summary of biological variables.

$121 \quad 2.3$ Construction of polygenic risk scores

$122 \quad$ 2.3.1 Overview of PRS model

123 According to the different phenotypes, we proposed to utilize multiple omics data and breast

124 cancer status to construct two kinds of PRS models. The first phenotype only contains the

125 normal samples (control) and tumor samples (case), which were labelled 0 and 1, respectively.

126 The second phenotype contains the normal samples, early-stage and late-stage tumor samples,

127 which were labelled 0,1 and 2, respectively. We defined the above-mentioned two PRS

128 models as PRS for case-control status and PRS for cancer stage status. The PRS can evaluate

129 the individual risk of breast cancer and may improve the diagnosis of breast cancer. Moreover,

130 since recent studies found the stage of cancer is highly associated with the prognosis[28],

131 accurate construction of PRS for cancer stage status may facilitate the prediction of breast

132 cancer prognosis. The framework of this study is shown in Figure 1. 


\subsubsection{PRS based on LightGBM}

134 LightGBM is an ensemble model of classification and regression trees (CART)[29], in which

135 each step generates a basic CART model and adds it to the overall model. The PRS models

136 based on LightGBM were built using a training dataset to predict PRS in a testing dataset. We

137 defined each omic dataset $D_{1}=\left\{\left(\mathbf{X}_{i}, y_{i}\right)\right\}\left(\left|D_{1}\right|=n_{1}, \mathbf{X}_{i} \in \mathbf{R}^{m}, y_{i} \in \mathbf{R}\right)$ as training dataset, where

$138 \mathbf{X}_{i}$ represents a matrix containing $n_{1}$ samples and $m$ biological variables, $y_{i}$ is the

139 corresponding outcome (phenotype). Let $\hat{y}_{i}$ be the prediction of $y_{i}$.

$140 D_{2}=\left\{\left(\mathbf{X}_{i}^{*}, y_{i}^{*}\right)\right\}\left(\left|D_{2}\right|=n_{2}, \mathbf{X}_{i}^{*} \in \mathbf{R}^{m}, y_{i}^{*} \in \mathbf{R}\right)$ was the testing dataset, where $\mathbf{X}_{i}^{*}$ represents a

141 matrix containing $n_{2}$ samples and $m$ biological variables, $y_{i}^{*}$ denotes the PRS. We used

$142 T$ additive CART models to predict the PRS in the training dataset.

$$
\hat{y}_{i}=\sum_{t=1}^{T} f_{t}\left(\mathbf{X}_{i}\right), f_{t} \in F
$$

144 where $f_{t}\left(\mathbf{X}_{i}\right)$ corresponds to an independent CART model and $F$ is the space of CART

145 models. To learn the set of CART used in the PRS model, we minimize the following 146 objective function.

$$
L\left(f_{t}\right)=\sum_{i=1}^{n_{1}} l\left(y_{i}, \hat{y}_{i}\right)+\gamma K+\frac{1}{2} \lambda \sum_{k=1}^{K} w_{k}^{2}
$$

148 Here $l\left(y_{i}, \hat{y}_{i}\right)$ is a differentiable convex loss function that measures the difference between

149 the prediction $\hat{y}_{i}$ and true phenotype $y_{i}$. The $K$ and $w_{k}$ respectively represent the 150 number and value of leaf nodes in each CART model, $\gamma$ and $\lambda$ are constant coefficients. 
151 In general setting, the second-order approximation can be utilized to quickly optimize the

152 objective function.

$$
L\left(f_{t}\right)=\sum_{k=1}^{K}\left[\left(\sum_{i \in I_{k}} g_{i}\right) w_{k}+\frac{1}{2}\left(\sum_{i \in I_{k}} h_{i}+\lambda\right) w_{k}^{2}\right]+\gamma K
$$

154 where $g_{i}$ and $h_{i}$ are the first and second order gradient statistics of the loss function.

$155 I_{k}=\left\{i \mid q\left(\mathbf{X}_{i}\right)=k\right\}$ was defined as the instance set of leaf node. LightGBM used two

156 techniques including gradient-based one-side sampling and exclusive feature bundling to

157 estimate the information gain in a high speed[21]. The structure and value of each CART

158 model can be determined by the information gain. Thus, we generated the PRS model

159 consisting of $T$ additive CART models. For the samples in a testing dataset, PRS $y_{i}^{*}$ can

160 be calculated by applying $\mathbf{X}_{i}^{*}$ to the PRS model. We also provided an automatic python

161 program based on our proposed models to obtain PRS, which is available for downloading

162 from GitHub website[30].

\subsubsection{PRS based on linear model and other ML model}

164 To evaluate the predictive performance of LightGBM objectively, we applied the traditional

165 linear model and other state-of-the-art ML models to construct PRS. The traditional linear

166 model contains minimax concave penalty (MCP)[17, 31], least absolute shrinkage and

167 selection operator (LASSO)[18, 32] and elastic net[33]. The ML model contains support

168 vector regression (SVR)[34]. Here, we compared the PRS methods that only utilize omics

169 data, without considering the methods that use GWAS summary statistics, such as 
LDpred[35], Lassosum[36] and so on. Similar to the PRS method based on LightGBM model,

171 we used each omic dataset as the input of these models, and the corresponding phenotypes as

172 the output.

\subsubsection{Model training and evaluation}

174 To ensure the robustness and stability of the model, we trained and evaluated the proposed

175 PRS model by 5-fold cross validation. This procedure divided each omic dataset into five

176 subsets. In each fold, one of the five subsets was used as the testing dataset and the other four

177 subsets were put together to form a training dataset. We applied bayesian optimization[37]

178 and 3-fold inner cross validation to optimize the hyper-parameters of the PRS model in each

179 training dataset. Specifically, for LASSO, we optimized the parameter "alpha". For MCP, we

180 adjusted regularization parameter "labmda". For elastic net, the parameter "alpha" and

181 "11_ratio" were optimized. For SVR, we choose "rbf kernel" and optimized the regularization

182 parameter "C". For LightGBM, the optimized parameters were "num_leaves", "n_estimators",

183 "learning_rate", "max_depth", "max_bin", "min_split_gain", "subsample", "subsample_freq",

184 "colsample_bytree", "min_child_sample", "min_child_weight", "reg_alpha", "reg_lambda".

185 Finally, we obtained the PRS of each testing dataset which was predicted by the model with

186 the optimized parameters. Each PRS was standardized based on its mean and standard

187 deviation. The predictive performance of PRS model was evaluated by square of the Pearson

188 correlation coefficient $\left(\mathrm{R}^{2}\right)$. 


$$
R^{2}=\left(\frac{\operatorname{Cov}(Y, \hat{Y})}{\sqrt{\operatorname{Var}(Y) \operatorname{Var}(\hat{Y})}}\right)^{2}
$$

190 where $\operatorname{Cov}(Y, \hat{Y})$ represents the covariation of true phenotype and predicted PRS, $\operatorname{Var}(Y)$

191 is the variance of true phenotype, and $\operatorname{Var}(\hat{Y})$ is the variance of predicted PRS. In addition,

192 for case-control status, we can also evaluate the predictive performance by the area under the

193 receiver operating characteristic curve (AUC).

\subsection{Combination model of PRS}

195 To further improve the predictive performance of PRS, we utilized the PRS based on each

196 omic dataset to construct a new combination model[38, 39]. We first matched a common

197 dataset from the four kinds of omics datasets for BRCA. In the common dataset of

198 case-control status, there are 786 tumor samples and 75 normal samples. In the common

199 dataset of cancer stage status, there are 553 early-stage samples, 205 late-stage samples and

20075 normal samples. Next, we used the PRS based on four kinds of omics datasets as new

201 biological variables for the combination model. Then, we build the combination model using

202 the LightGBM model. Bayesian optimization was applied to adjust hyper-parameters and

2035 -fold cv was used to evaluate the overall predictive performance. The framework of the

204 combination model is shown in Supplementary Figure 2.

\section{3. Results}

\subsection{Predictive performance of PRS based on multiple omics data}


207 We first compared our prediction model to existing PRS methods and other ML methods for 208 case-control status. Figure 2a shows the results of these PRS methods on four kinds of omics 209 datasets. We observed that elastic net achieves the best performance in traditional linear 210 models. The $\mathrm{R}^{2}$ of SVR is $3.3 \%, 7.7 \%$ and $0.5 \%$ higher than elastic net on DNA methylation, 211 miRNA and lncRNA datasets and 3.1\% lower than elastic net on mRNA dataset. The $\mathrm{R}^{2}$ of 212 our proposed model improved by $8.3 \%, 14.8 \%, 5.1 \%$ and $7.2 \%$ than elastic net on four kinds 213 of omics datasets. Overall, our model outperformed other models and mRNA data exhibited 214 better performance than other omics data.

215 Next, we applied our proposed model and other PRS methods for cancer stage status.

216 Compared with the case-control status, this phenotype contains normal and two stage statuses

217 of breast cancer. Thus, the predictive performance of PRS for cancer stage status is not as 218 good. Nevertheless, the present results are consistent with the PRS for case-control status. 219 According to the comparison results of our proposed model with other PRS methods (Figure 220 2b), the LightGBM model performs the best predictive performance, outscoring other PRS 221 methods on four kinds of omics datasets. The PRS based on LightGBM obtains the $\mathrm{R}^{2}$ of $2220.405,0.371,0.437$ and 0.407 , respectively. Compared with the elastic net with the highest 223 PA in the linear models, the $\mathrm{R}^{2}$ of LightGBM improved by $12.8 \%, 20.9 \%, 9.8 \%$ and $12.4 \%$, 224 respectively. Compared with SVR, the $\mathrm{R}^{2}$ of our proposed model improved by $10.4 \%, 14.4 \%$, $22510.6 \%$ and 3.3\%, respectively. Moreover, the results showed mRNA data obtained better 226 results than other omics data. 
In this part, we evaluated the performance of combination model of PRS. Figure $2 \mathrm{c}$ shows the

229 results of PRS models based on four types of omics datasets and combination model in the

230 common samples. For four kinds of omics datasets, although the PA in the common samples

231 has decreased, we found that PRS based on mRNA still obtained the best PA. For

232 case-control and cancer stage status, the $\mathrm{R}^{2}$ of combination model were 0.932 and 0.397 ,

233 respectively. Compared with the PRS model based on mRNA dataset, the $\mathrm{R}^{2}$ of combination

234 model improved by $5.1 \%$ and $2.8 \%$, respectively. Thus, the combination of four types of

235 molecular data can achieve better results of PRS for case-control and cancer stage status.

\section{$236 \quad 3.3$ Prevalence of breast cancer}

237 Exploring the prevalence of different PRS strata has a positive impact on the prevention and 238 treatment of breast cancer[40]. The main goal of this part is to analyze the risk stratification of 239 case-control status. Thus, we divided the common samples into 10 strata of increasing PRS

240 from the combination model and calculated the prevalence of each stratum (Figure 3).

241 Across the common samples, we observed that the prevalence is about $10 \%$ in the first

242 stratum then upgrades to $100 \%$ in the second stratum and remains steady afterwords. The

243 prevalence changes significantly at one stratum because our proposed method achieved 244 relatively accurate prediction of breast cancer risk for case-control status. The trend plot of the 245 prevalence also indicated that individuals with high-PRS strata have greater breast cancer risk 246 than the individuals with lower-PRS strata.

\section{3.4 Associations between PRS and breast cancer risk}


248 We investigated the relationship of PRS with different phenotypes of breast cancer in this

249 section (Table 2). For case-control status, the association of PRS was evaluated in predicted

250 results from the combination model by Logistic regression. We observed that PRS was

251 associated with occurrence risk of breast cancer (odds ratio $(\mathrm{OR})=18.48 ; 95 \%$ confidence

252 interval $\left.(\mathrm{CI}): 9.60-35.55 ; \mathrm{P}=2.46 \times 10^{-18}\right)$, suggesting that per one standard deviation increase

253 in PRS is associated with $170 \%$ risk increase of breast cancer. For cancer stage status, we

254 performed a multinomial Logistic regression model to evaluate the association of PRS and set

255 the normal sample as the reference group. The PRS was associated with early-stage breast

256 cancer risk $\left(\mathrm{OR}=21.05 ; 95 \% \mathrm{CI}: 10.26-43.19 ; \mathrm{P}=9.63 \times 10^{-17}\right)$ and late-stage breast cancer

257 risk $\left(\mathrm{OR}=46.62 ; 95 \% \mathrm{CI}: 19.72-110.25 ; \mathrm{P}=2.14 \times 10^{-18}\right)$. The results indicated higher PRS is

258 associated with a significantly increased risk for early-stage and late-stage breast cancer.

Table 2. Associations between PRS and breast cancer risk.

\begin{tabular}{|c|c|c|c|c|}
\hline \multicolumn{2}{|c|}{ Phenotype } & OR & $95 \%$ CI & P-value \\
\hline \multicolumn{2}{|c|}{ The PRS for case-control status } & 18.48 & $9.60-35.55$ & $2.46 \times 10^{-18}$ \\
\hline $\begin{array}{l}\text { The PRS for } \\
\text { cancer stage }\end{array}$ & Early-stage & 21.05 & $10.26-43.19$ & $9.63 \times 10^{-17}$ \\
\hline status & Late-stage & 46.62 & $19.72-110.25$ & $2.14 \times 10^{-18}$ \\
\hline
\end{tabular}

259

PRS: Polygenic risk score; OR: Odds ratio; CI: Confidence intervals.

\subsection{Prognosis prediction of breast cancer}

261 We explored whether the PRS for cancer stage status can effectively assess the prognosis of

262 patients. According to the predicted results of tumor samples using combination model, we

263 firstly divided 758 patients of breast cancer into high-risk and low-risk groups based on the 
264 50th percentile of PRS. Next, we utilized the survival time and the status at the end of their

265 survival time for each patient to generate Kaplan-Meier curves (KM curve)[41]. We observed

266 that high-risk patients had statistically significantly worse prognosis (Figure 4). The results

267 showed the PRS for cancer stage may provide an effective prognostic tool of breast cancer 268 patients.

\section{4. Discussion}

270 In our study, first of all, we have developed a novel PRS method for breast cancer using

271 multiple omics data and LightGBM model. For case-control and cancer stage status, we

272 showed that the proposed method had better prediction performance than existing traditional

273 linear models and state-of-art ML models using multiple omics data. Meanwhile, the

274 prediction results of 5-fold-cv demonstrated the robustness and reliability of our proposed

275 method. Second, the combination of PRS further improved the predictive performance for

276 breast cancer. Finally, by analyzing the trend of prevalence and associations between PRS and

277 breast cancer risk, the results bolstered the clinical understanding and application for breast

278 cancer PRS. In addition, we also found that our PRS models for cancer stage status can

279 improve the prognosis prediction of breast cancer patients.

280 Most of the previous PRS studies focused on the analysis of individual-level genotype data

281 (SNPs) using linear models. For example, Mavaddat et al. utilized PRS derived from 313

282 SNPs in 69 studies of the Breast Cancer Association Consortium (BCAC) to predict the breast

283 cancer risk and the AUC was 0.63[9]. Khera et al. derived a PRS based on 5218 SNPs in the 
284 UK Biobank and the AUC was 0.68[8]. Although these studies obtain individual-level genetic 285 risk of breast cancer, the current PA still maintains at low level. In case-control status of our 286 study, we obtained the AUC of 0.99 from the combination model. Thus, the PRS based on 287 multiple omics data and LightGBM not only improved the risk of predicting breast cancer, 288 but also expanded the current definition of PRS from SNP data to genomics and 289 transcriptomics data.

290 Our proposed method outperforms the other breast cancer PRS for two main reasons. First,the 291 LightGBM model fits all biological variables simultaneously using gradient boosting tree, 292 especially high-dimensional data such as multiple omics data[21], while linear model such as 293 MCP, LASSO only utilizes marginal variables to construct PRS. In addition, the LightGBM 294 model takes advantage of ensemble learning, which helps to minimize the main causes of 295 error in ML model such as noise, bias and variance than a single model[42]. Second, as 296 representative of genomics data, DNA methylation can be modulated by physiological and 297 environmental exposures and provided biomarkers for diagnosis and prognosis for cancer[43, 298 44]. Transcriptomics data including miRNA, mRNA, and lncRNA reveals the transcription 299 and regulation mechanism of large-scale genes, which play an important role in determining 300 the mechanism and treatment of cancer[45, 46]. Compared to the individual-level genotype 301 data, using multiple omics data to construct breast cancer PRS considered the interaction of 302 genetic and environmental factors, and thus can provide higher PA. 
303 Although our PRS methods provide powerful predictive performance, they have some

304 limitations. First, the LightGBM model has more hyper-parameters than traditional linear 305 models such as MCP, LASSO and elastic net. Thus, we need more time to train the proposed 306 model. We applied multithreading technology to effectively utilize computing resources and 307 correspondingly reduced some running time. Second, the sample size of breast cancer from 308 TCGA is relatively small compared to large-scale Genome-wide association studies data. In 309 addition, there are significantly more tumor samples than normal samples in our study. 310 Imbalanced datasets significantly compromise the performance of most standard learning 311 algorithms, because these models assume the balanced class distributions. Third, this study 312 lacks independent validation datasets, because it is very difficult to collect multiple omics 313 data including DNA methylation, miRNA, mRNA and lmcRNA of case-control and cancer 314 stage status. Thus, we employed 5-fold cross validation to strengthen the robustness and 315 stability of our proposed models. In the future, we will consider applying our PRS model to 316 analyze breast cancer with other phenotypes by using larger and balanced multiple omics 317 datasets.

\section{5. Conclusions}

319 In conclusion, we proposed a novel PRS model in two kinds of breast cancer phenotypes by 320 using multiple omics data and LightGBM. The results demonstrated our model improved the

321 PA of current PRS methods indeed and may provide an effective diagnosis and prognosis tool

322 for breast cancer. 
324 GWAS: Genome-wide association study; PRS: Polygenic risk score; SNPs: Single nucleotide 325 polymorphisms; ML: Machine learning; PA: Prediction accuracy; TCGA: The Cancer 326 Genome Atlas; BRCA: Breast invasive carcinoma; CART: Classification and regression trees; 327 MCP: Minimax concave penalty; LASSO: Least absolute shrinkage and selection operator; 328 SVR: Support vector regression; AUC: Area under the receiver operating characteristic curve; 329 OR: Odds ratio; CI: Confidence interval; KM: Kaplan-Meier; BCAC: Breast Cancer 330 Association Consortium

\section{Declarations}

\section{Ethics approval and consent to participate}

333 All TCGA data are accessible without limitations in publications or presentations according to 334 the posted announcement from the TCGA website.

\section{Consent for publication}

336 All authors give consent to the publication.

\section{Availability of data and materials}

338 The datasets used during the current study are available from the TCGA website

339 (https://portal.gdc.cancer.gov/).

\section{Competing Interests}

341 The authors declare no competing financial interests. 


\section{Funding}

343 This work was supported by the National Natural Science Foundation of China (61471078),

344 Dalian Science and Technology Innovation Fund (2020JJ27SN066) and the Fundamental

345 Research Funds for the Central Universities (3132014306,3132015213, 3132017075).

\section{Authors' contributions}

347 Supervision: BSM and FJS. Study design: BSM, FJS and JQP. Data Collection: JQP, TX and

348 JCL. Software \& analysis: JQP, CYL and XYH. Writing-original draft: JQP. Editing: BSM,

349 FJS, and JQP. Writing-review: All authors.

\section{Acknowledgements}

$351 \quad$ Not applicable

\section{References}

1. Britt KL, Cuzick J, Phillips K-A. Key steps for effective breast cancer prevention. Nature Reviews Cancer. 2020;20(8):417-436.

2. Wild C, Weiderpass E, Stewart B. World cancer report: cancer research for cancer prevention. Lyon: International Agency for Research on Cancer. 2020:23-33.

3. Thompson D, Easton D. The genetic epidemiology of breast cancer genes. Journal of mammary gland biology and neoplasia. 2004;9(3):221-236.

4. Wu L, Shi W, Long J, Guo X, Michailidou K, Beesley J, et al. A transcriptome-wide association study of 229,000 women identifies new candidate susceptibility genes for breast cancer. Nature genetics. 2018;50(7):968-978.

5. Gail MH. Discriminatory accuracy from single-nucleotide polymorphisms in models to predict breast cancer risk. Journal of the National Cancer Institute. 2008;100(14):1037-1041.

6. Maas P, Barrdahl M, Joshi AD, Auer PL, Gaudet MM, Milne RL, et al. Breast cancer risk from modifiable and nonmodifiable risk factors among white women in the United States. JAMA oncology. 2016;2(10):1295-1302. 
7. Mavaddat N, Pharoah PD, Michailidou K, Tyrer J, Brook MN, Bolla MK, et al. Prediction of breast cancer risk based on profiling with common genetic variants. JNCI: Journal of the National Cancer Institute. 2015;107(5).

8. Khera AV, Chaffin M, Aragam KG, Haas ME, Roselli C, Choi SH, et al. Genome-wide polygenic scores for common diseases identify individuals with risk equivalent to monogenic mutations. Nature genetics. 2018;50(9):1219-1224.

9. Mavaddat N, Michailidou K, Dennis J, Lush M, Fachal L, Lee A, et al. Polygenic risk scores for prediction of breast cancer and breast cancer subtypes. The American Journal of Human Genetics. 2019;104(1):21-34.

10. Dor Y, Cedar H. Principles of DNA methylation and their implications for biology and medicine. The Lancet. 2018;392(10149):777-786.

11. Lowe R, Shirley N, Bleackley M, Dolan S, Shafee T. Transcriptomics technologies. PLoS computational biology. 2017;13(5):e1005457.

12. Wild CP. Complementing the genome with an "exposome": the outstanding challenge of environmental exposure measurement in molecular epidemiology. Cancer Epidemiology and Prevention Biomarkers. 2005;14(8):1847-1850.

13. Alegría-Torres JA, Baccarelli A, Bollati V. Epigenetics and lifestyle. Epigenomics. 2011;3(3):267-277.

14. Wild CP. The exposome: from concept to utility. International journal of epidemiology. 2012;41(1):24-32.

15. Sun YV, Hu Y-J. Integrative analysis of multi-omics data for discovery and functional studies of complex human diseases. Advances in genetics. 2016;93:147-190.

16. Speed D, Balding DJ. MultiBLUP: improved SNP-based prediction for complex traits. Genome research. 2014;24(9):1550-1557.

17. Liu J, Wang K, Ma S, Huang J. Accounting for linkage disequilibrium in genome-wide association studies: A penalized regression method. Statistics and its interface. 2013;6(1):99.

18. Lello L, Avery SG, Tellier L, Vazquez AI, de Los Campos G, Hsu SD. Accurate genomic prediction of human height. Genetics. 2018;210(2):477-497.

19. Choi SW, Mak TS-H, O'Reilly PF. Tutorial: a guide to performing polygenic risk score analyses. Nature Protocols. 2020;15(9):2759-2772.

20. Erenpreisa J, Giuliani A. Resolution of complex issues in genome regulation and cancer requires non-linear and network-based thermodynamics. International journal of molecular sciences. 2020;21(1):240.

21. Ke G, Meng Q, Finley T, Wang T, Chen W, Ma W, et al. Lightgbm: A highly efficient gradient boosting decision tree. Advances in neural information processing systems. 2017;30:3146-3154.

22. Zhu E, Jiang F, Liu C, Xu J. Partition Independent Set and Reduction-Based Approach for Partition Coloring Problem. IEEE Transactions on Cybernetics. 2020. doi: 10.1109/TCYB.2020.3025819.

23. Tomczak K, Czerwińska P, Wiznerowicz M. The Cancer Genome Atlas (TCGA): an immeasurable source of knowledge. Contemporary oncology. 2015;19(1A):A68. 
24. Rahimi A, Gönen M. Discriminating early-and late-stage cancers using multiple kernel learning on gene sets. Bioinformatics. 2018;34(13):i412-i421.

25. Yuan Y, Van Allen EM, Omberg L, Wagle N, Amin-Mansour A, Sokolov A, et al. Assessing the clinical utility of cancer genomic and proteomic data across tumor types. Nature biotechnology. 2014;32(7):644-652.

26. Liu B, Liu Y, Pan X, Li M, Yang S, Li SC. DNA methylation markers for pan-cancer prediction by deep learning. Genes. 2019;10(10):778.

27. Ma B, Meng F, Yan G, Yan H, Chai B, Song F. Diagnostic classification of cancers using extreme gradient boosting algorithm and multi-omics data. Computers in biology and medicine. 2020;121:103761.

28. Weiss A, Chavez-MacGregor M, Lichtensztajn DY, Yi M, Tadros A, Hortobagyi GN, et al. Validation study of the American Joint Committee on Cancer eighth edition prognostic stage compared with the anatomic stage in breast cancer. JAMA oncology. 2018;4(2):203-209.

29. De'ath G, Fabricius KE. Classification and regression trees: a powerful yet simple technique for ecological data analysis. Ecology. 2000;81(11):3178-3192.

30. Pan J. The python program of PRS based on multiple omics data and LightGBM. https://github.com/lab319/PRS BRCA omics LightGBM Accessed 11 Feb 2021.

31. Zhang C-H. Nearly unbiased variable selection under minimax concave penalty. The Annals of statistics. 2010;38(2):894-942.

32. Tibshirani R. Regression shrinkage and selection via the lasso. Journal of the Royal Statistical Society: Series B (Methodological). 1996;58(1):267-288.

33. Zou H, Hastie T. Regularization and variable selection via the elastic net. Journal of the royal statistical society: series B (statistical methodology). 2005;67(2):301-320.

34. Smola AJ, Schölkopf B. A tutorial on support vector regression. Statistics and computing. 2004;14(3):199-222.

35. Vilhjálmsson BJ, Yang J, Finucane HK, Gusev A, Lindström S, Ripke S, et al. Modeling linkage disequilibrium increases accuracy of polygenic risk scores. The american journal of human genetics. 2015;97(4):576-592.

36. Mak TSH, Porsch RM, Choi SW, Zhou X, Sham PC. Polygenic scores via penalized regression on summary statistics. Genetic epidemiology. 2017;41(6):469-480.

37. Snoek J, Larochelle H, Adams RP, editors. Practical Bayesian optimization of machine learning algorithms. 26th Annual Conference on Neural Information Processing Systems 2012, NIPS 2012; 2012.2951-2959

38. Alves A. Stacking machine learning classifiers to identify Higgs bosons at the LHC. Journal of Instrumentation. 2017;12(05):T05005.

39. Pavlyshenko B, editor Using stacking approaches for machine learning models. 2018 IEEE Second International Conference on Data Stream Mining \& Processing (DSMP); 2018: IEEE.255-258

40. Barendregt JJ, Doi SA, Lee YY, Norman RE, Vos T. Meta-analysis of prevalence. J Epidemiol Community Health. 2013;67(11):974-978. 
41. Rich JT, Neely JG, Paniello RC, Voelker CC, Nussenbaum B, Wang EW. A practical guide to understanding Kaplan-Meier curves. Otolaryngology—Head and Neck Surgery. 2010;143(3):331-336.

42. Polikar R. Ensemble learning. Ensemble machine learning: Springer; 2012. p. 1-34.

43. Pan Y, Liu G, Zhou F, Su B, Li Y. DNA methylation profiles in cancer diagnosis and therapeutics. Clinical and experimental medicine. 2018;18(1):1-14.

44. Cheng Y, He C, Wang M, Ma X, Mo F, Yang S, et al. Targeting epigenetic regulators for cancer therapy: Mechanisms and advances in clinical trials. Signal transduction and targeted therapy. 2019;4(1):1-39.

45. Fan J, Slowikowski K, Zhang F. Single-cell transcriptomics in cancer: Computational challenges and opportunities. Experimental \& Molecular Medicine. 2020;52(9):1452-1465.

46. Rodon J, Soria J-C, Berger R, Miller WH, Rubin E, Kugel A, et al. Genomic and transcriptomic profiling expands precision cancer medicine: the WINTHER trial. Nature medicine. 2019;25(5):751-758. 


\section{Figure Legends}

482

483

484

\section{Figure 1. Schematic overview of the framework for constructing PRS model based on} multiple omics data. The dataset of BRCA was split into two groups as training dataset and testing dataset based on 5-fold cross validation. We constructed PRS model by using MCP, LASSO, elastic net, SVM and LightGBM based on training dataset. The hyper-parameters of five models were optimized by using bayesian optimization and 3-fold cross validation. The PRS of testing dataset was predicted by optimized model. The predictive performance of final models was evaluated with $\mathrm{R}^{2}$.

Figure 2. Predictive performance of MCP, LASSO, elastic net, SVR and LightGBM in four kinds of omics datasets. (A) Comparison results of multiple omics datasets for case-control status. (B) Comparison results of multiple omics datasets for cancer stage status. (C) Comparison results of multiple omics datasets and combination model in the common samples for case-control and cancer stage status.

Figure 3. Prevalence strata plot of increasing PRS for case-control status. The sample size of 10 strata was equal and the prevalence of BRCA increased with the increase of PRS. The 1st stratum can be regarded as a low-risk PRS stratum and the 2nd to 10th stratum as a high-risk stratum.

\section{Figure 4. The KM survival curve of BRCA patients in the high-risk and low-risk} groups. We divided patients into high-risk and low-risk groups based on the 50th PRS. The patients with low-risk group have better prognosis than those with high-risk group. 


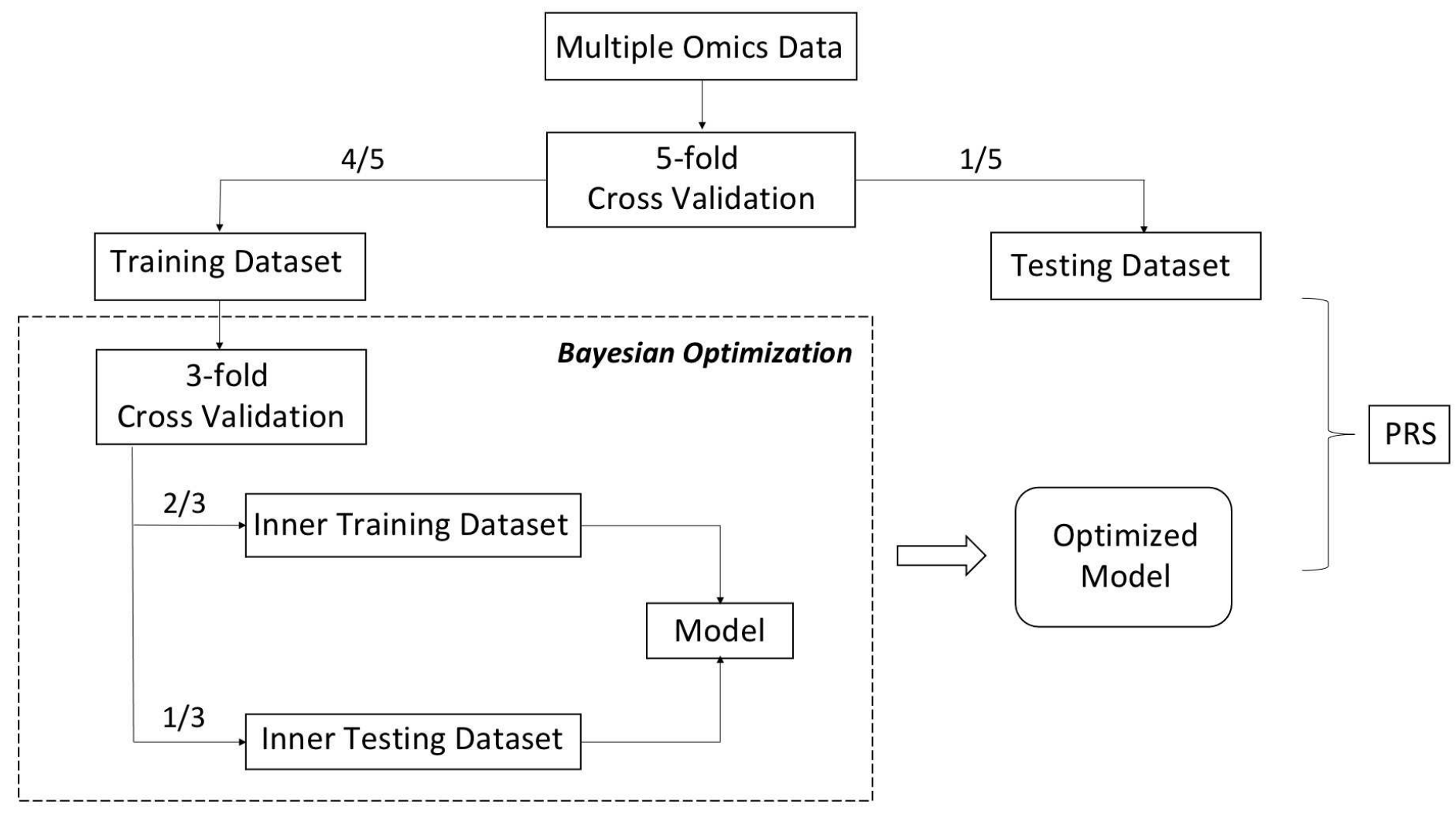




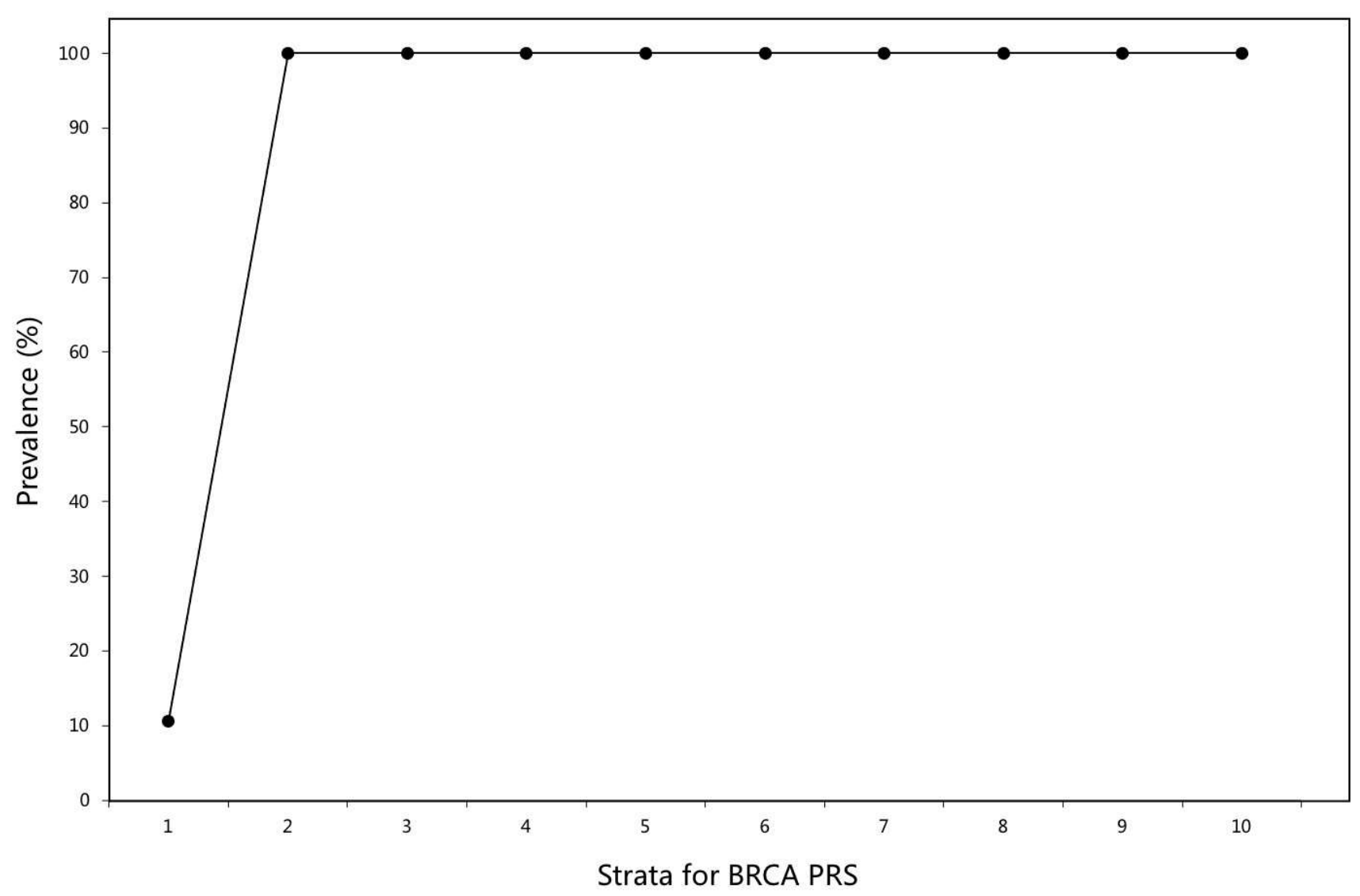




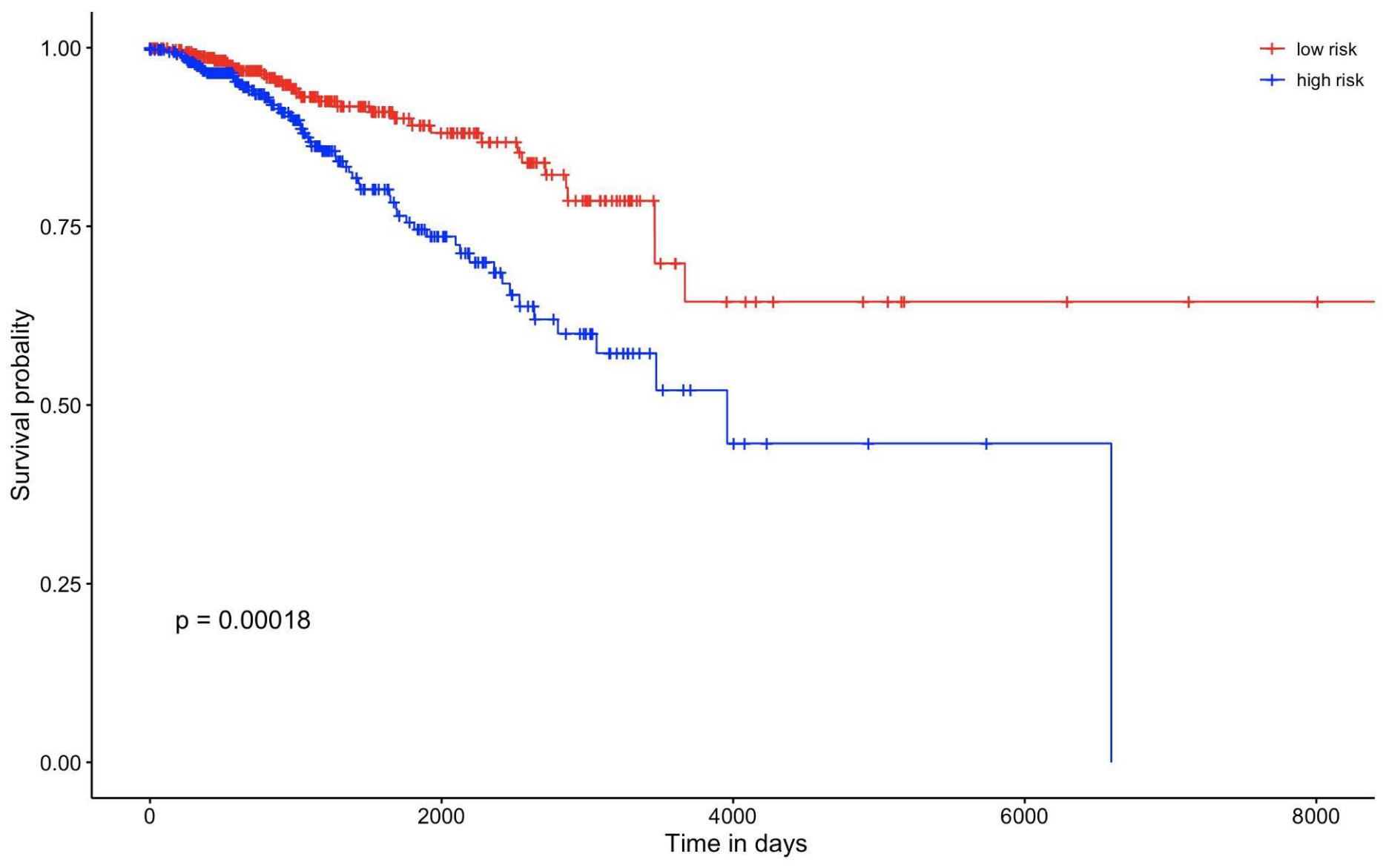


A

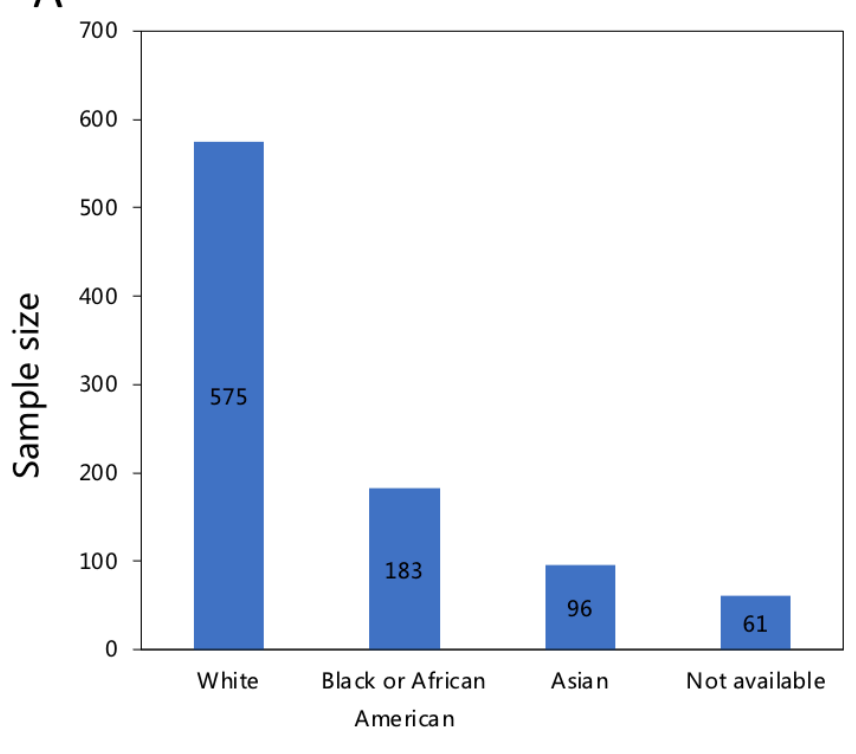

C

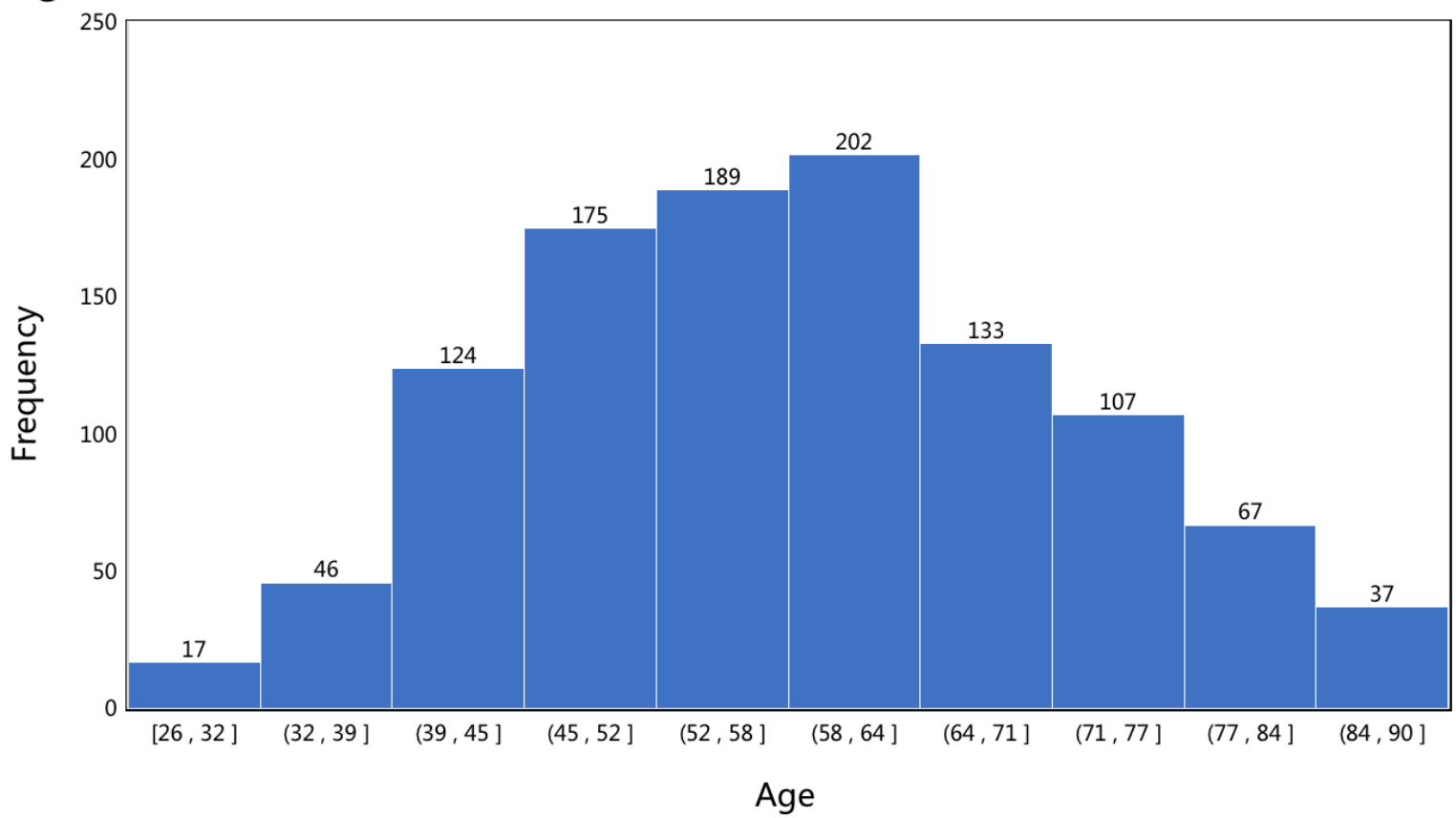

B

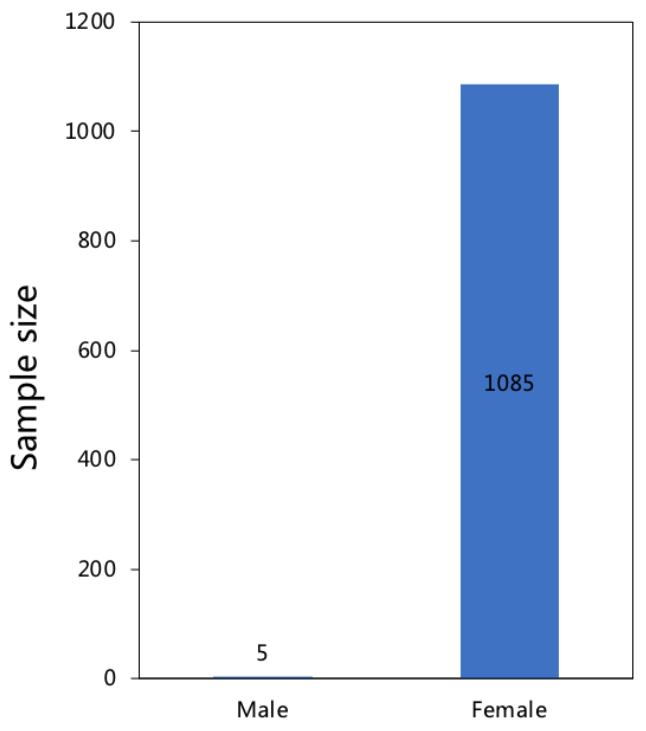

Gender

Supplementary Figure 1. Statistics of basic demographic characteristics of patients in BRCA datasets.

(A) Sample size of race including white, Black or African American, Asian and not available. (B) Sample size of 


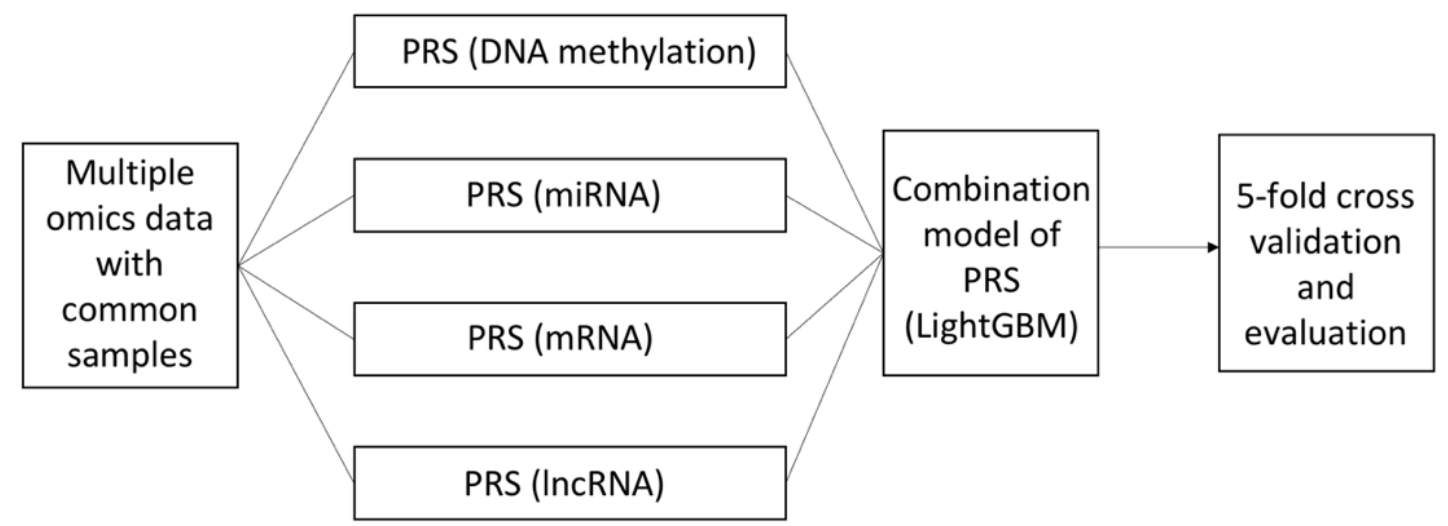

Supplementary Figure 2. Schematic overview of the framework for constructing combination model of

PRS. We utilized proposed PRS model based on each omic data (DNA methylation, miRNA, mRNA and lncRNA) as new biological variables. The combination model was constructed by LightGBM model. The hyper-parameters of combination model were optimized by using bayesian optimization and 3-fold cross validation. The predictive performance was evaluated by 5 -fold cross validation. 


\section{Figures}

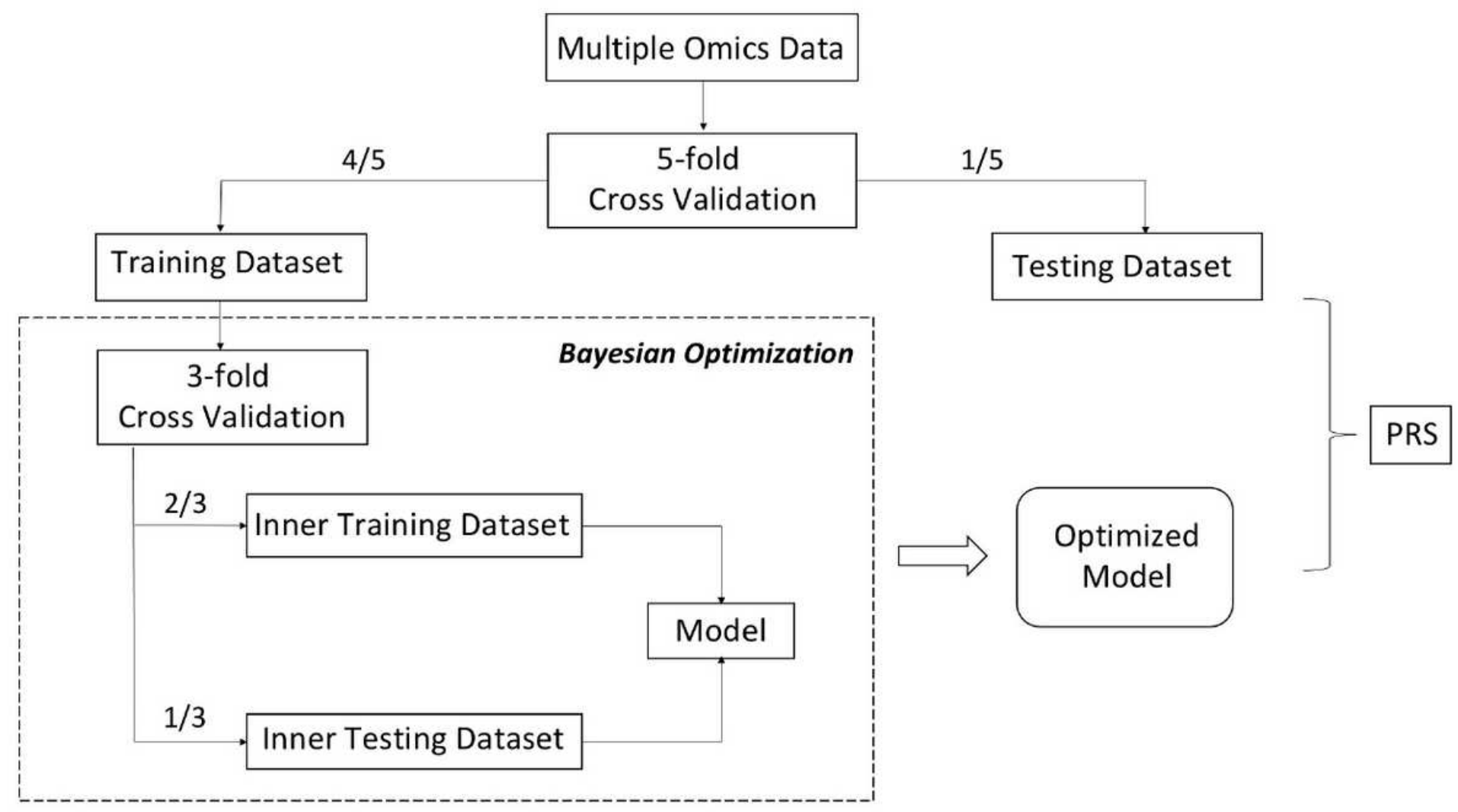

Figure 1

Schematic overview of the framework for constructing PRS model based on multiple omics data. The dataset of BRCA was split into two groups as training dataset and testing dataset based on 5 -fold cross validation. We constructed PRS model by using MCP, LASSO, elastic net, SVM and LightGBM based on training dataset. The hyper-parameters of five models were optimized by using bayesian optimization and 3-fold cross validation. The PRS of testing dataset was predicted by optimized model. The predictive performance of final models was evaluated with R2. 


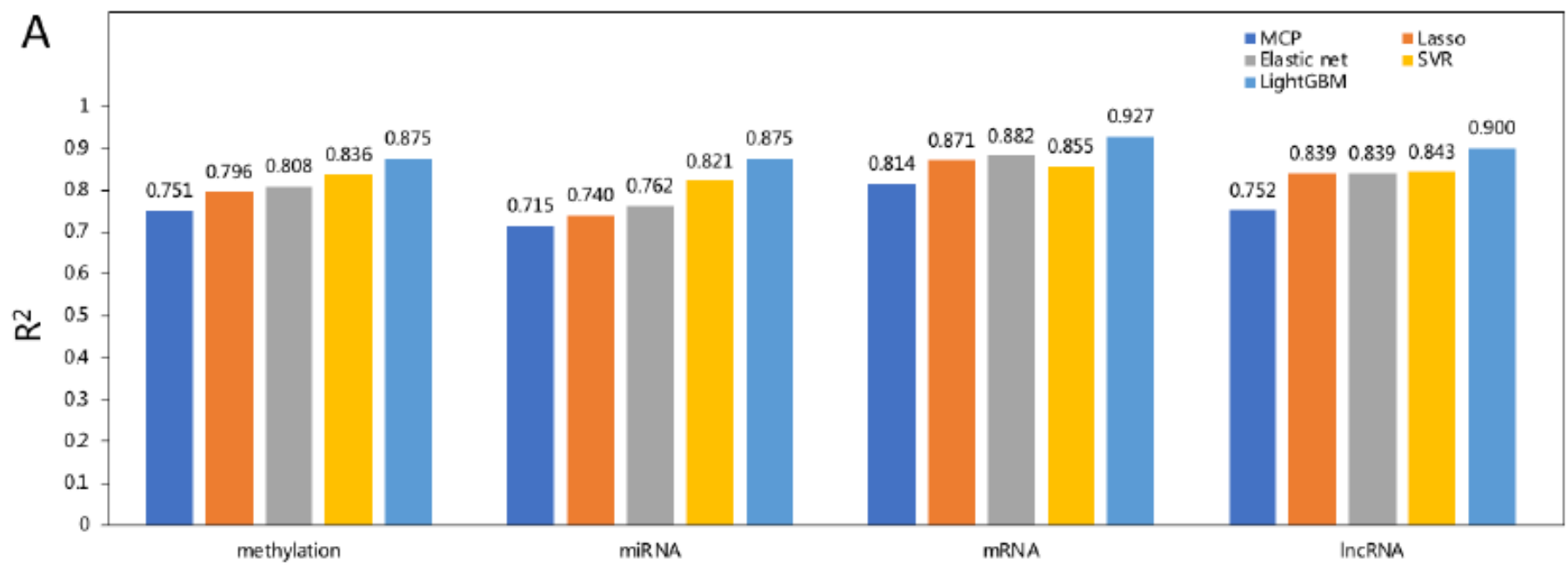

B

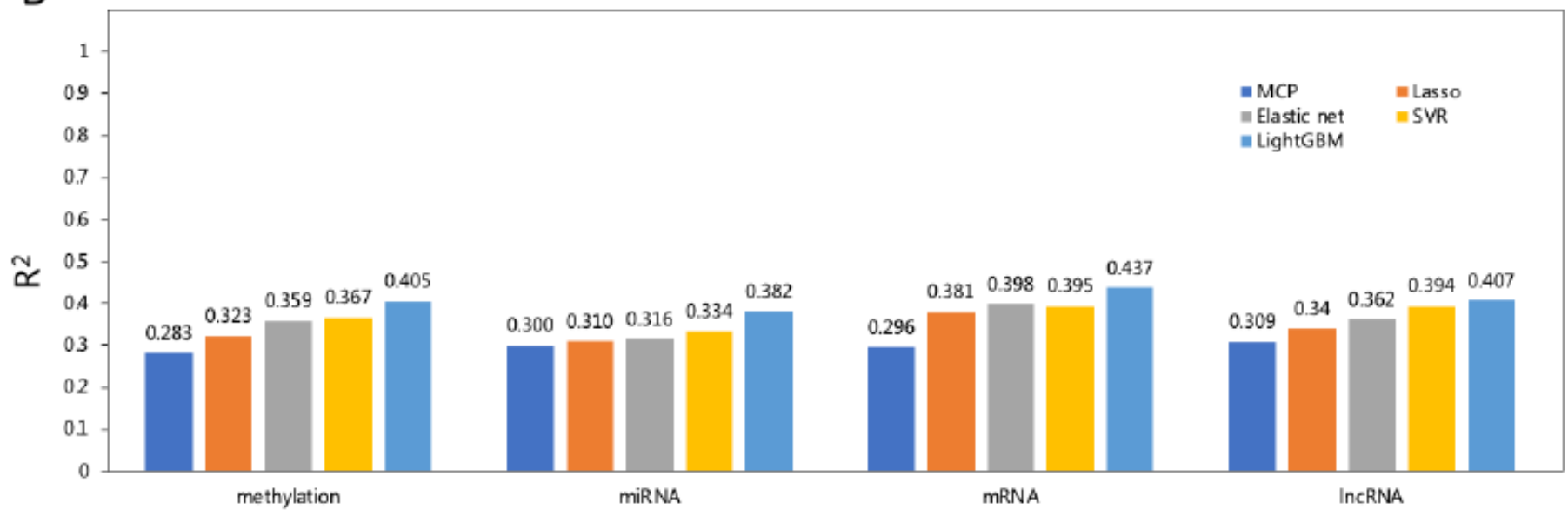

C

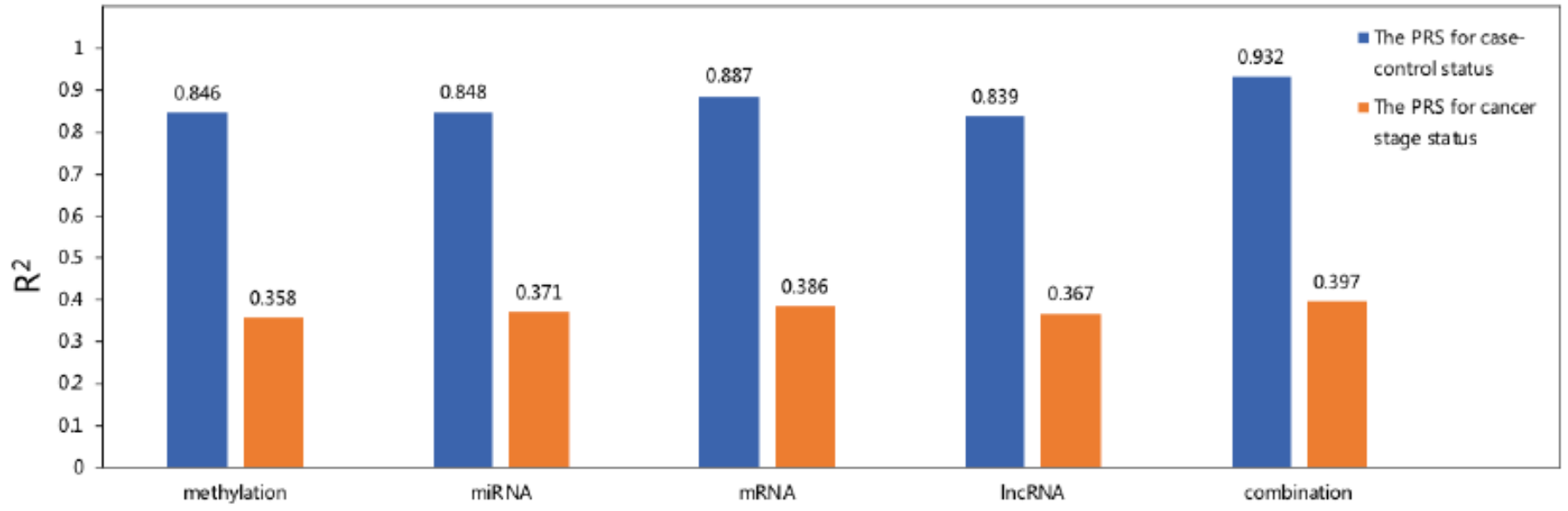

Figure 2

Predictive performance of MCP, LASSO, elastic net, SVR and LightGBM in four kinds of omics datasets. (A) Comparison results of multiple omics datasets for case-control status. (B) Comparison results of multiple omics datasets for cancer stage status. (C) Comparison results of multiple omics datasets and combination model in the common samples for case-control and cancer stage status. 


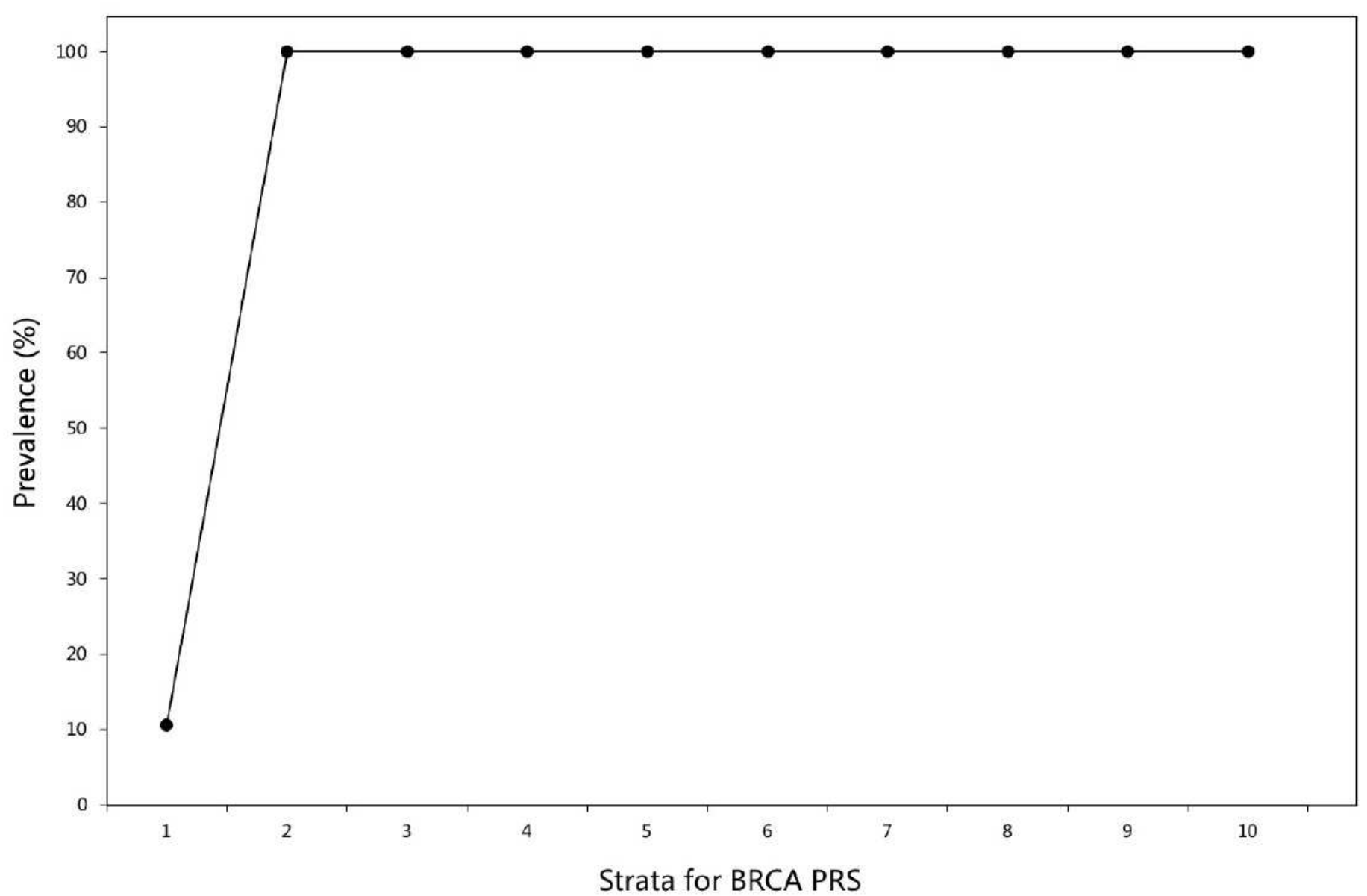

Figure 3

Prevalence strata plot of increasing PRS for case-control status. The sample size of 10 strata was equal and the prevalence of BRCA increased with the increase of PRS. The 1st stratum can be regarded as a low-risk PRS stratum and the 2 nd to 10 th stratum as a high-risk stratum. 


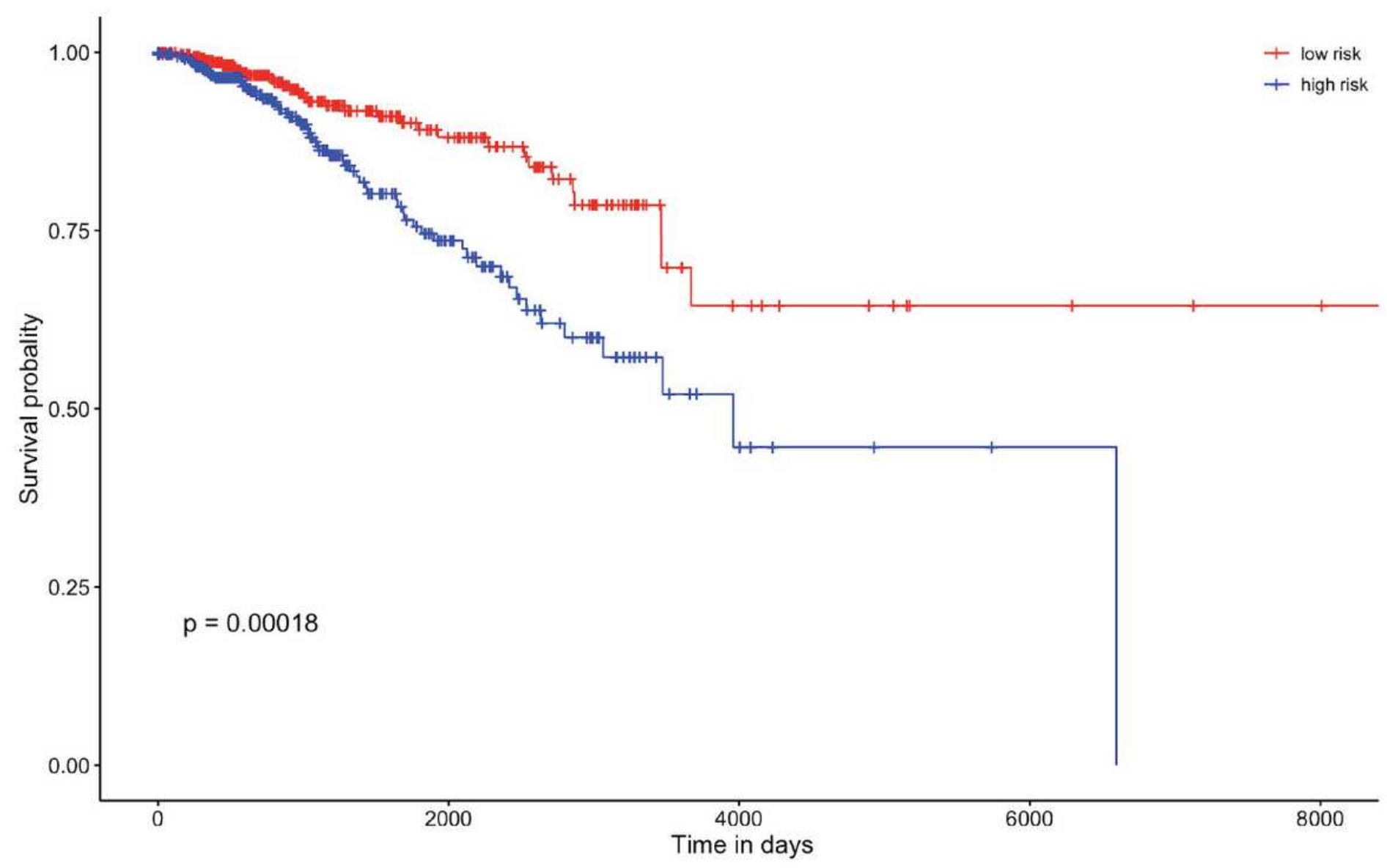

Figure 4

The KM survival curve of BRCA patients in the high-risk and low-risk groups. We divided patients into high-risk and low-risk groups based on the 50th PRS. The patients with low-risk group have better prognosis than those with high-risk group. 